\title{
Heart Rates of Participants in Wheelchair Sports
}

\author{
K. D. Coutts, PhD \\ Department of Sport Sciences, University of British Columbia, 6081 University \\ Blvd., Vancouver, BC, Canada, V6T $1 \mathrm{W5}$
}

\begin{abstract}
Summary
The relative stress of participation in wheelchair basketball, volleyball, tennis, and racquetball were determined by monitoring the heart rates of wheelchair athletes. Heart rates were recorded for 5 seconds every 30 seconds during monitoring sessions of 10 min or longer under game or practice conditions. Subjects were volunteer paraplegic athletes with lesions below $T 5$ or with equivalent disability according to an international sport classification system. Average heart rates were 89 beats/min for tennis 'practice', 96 for racquetball 'practice', 107 for volleyball 'practice', 114 for volleyball 'game', 127 for tennis 'game', 129 for basketball 'practice', 135 for racquetball 'game', and 149 for basketball 'game' conditions. The percentage of time when heart rates were above 140 beats/min, followed the same pattern as the average heart rates and ranged from 0 to $62 \%$.
\end{abstract}

Key words: Paraplegics; Wheelchair sports; Heart rates.

The physical fitness benefits of participation in wheelchair sports, have not been extensively studied. Hjeltnes and Vokac (1979) noted average daily heart rates of 76 to 101 beats/min in a group of five non-hospitalised and one hospitalised paraplegic. Heart rates greater than $50 \%$ of the individual's heart rate reserve $(50 \%$ of the difference between resting and maximal heart rate) occurred only during ambulation with crutches, uphill wheelchair propulsion, wheelchair basketball, or a specific arm crank training program in these subjects. Playing wheelchair table tennis produced peak heart rates of 111 to 115 beats/min in three of their subjects, which was below the $50 \%$ of heart rate reserve they deemed necessary for circulatory fitness benefits. In a study during the XXI International Stoke Mandeville Games, Hullemann, List, Mathes, Weise, and Zika (1975), noted heart rates above 180 beats per minute in male and female competitors during track and swimming events and below 130 during archery and field events. A more recent study by Mollinger, et al. (1985) noted average daily heart rates of 87,90 , and 90 beats/min in groups of post-rehabilitation low 
lesion quadriplegics, high lesion paraplegics, and low lesion paraplegics, respectively, during hospitalisation for routine medical evaluation. Individual average heart rates ranged from 69 to 136 beats $/ \mathrm{min}$, and there was no breakdown of the daily average by type of activity.

The purpose of this paper is to describe the heart rates of participants in a number of wheelchair sports, which will provide information on the intensity of the activities and thus indicate possible cardiovascular fitness benefits from participation in these activities.

\section{Methods}

An incidental, volunteer sample of 18 male participants in wheelchair sports in the greater metropolitan areas of Vancouver, and Victoria, British Columbia served as subjects. Written informed consent was obtained from each subject, and a brief history to determine cause and level of disability was taken. All subjects were living in non-institutional settings and participated in regular team or individual practices and competitions. Most of the subjects were disabled due to traumatic spinal cord injury, but the sample included individuals disabled due to poliomyelitis, spina bifida, spinal tumour, and lower limb amputation. Subjects included in the data analysis were limited to those who presented histories or had previously been classified for sport competitions that placed them in classes III, IV, or V, according to the International StokeMandeville Games classification system (Biering-Sorensen, 1980). This limitation was placed on the sample due to a previous finding that subjects with lesions above T6 may have reduced maximal exercise heart rates as a result of partial loss of sympathetic cardiac control (Coutts, Rhodes and McKenzie, 1983). The subjects ranged in age from 18 to 48 years old with an average age of 28.

The heart rates of the subjects were monitored over at least a 10 minute consecutive period of activity in a wheelchair sport. The sports monitored included basketball, volleyball, tennis and racquetball. The monitoring was further broken down into 'practice' or 'game' categories within each activity. The 'game' category did not include games that were part of any recognised league or tournament, but were intra or inter-team or individual games or scrimmages. These 'games' formed a regular part of the training for actual competition, and the recorded heart rates would thus reflect the athlete's exposure to this intensity of activity on a regular basis rather than the more infrequent actual competitions. The basketball and volleyball 'games' were under full court and team complement conditions with a referee to control play and scores were recorded. In the case of racquetball and tennis, all 'games' were singles matches with the rule that the ball could hit the floor or ground twice before being played by the opponent. These matches were refereed by the players with normal scoring procedures. Only one player was monitored at a time to avoid possible interference of the transmitted signals. Some subjects were monitored during more than one sport, but only if they were regular participants in the activity. Thus, each subject was reasonably skilled in the sport or activity being monitored. This was considered an appropriate limitation on the data collection since it eliminated the heart rates of subjects performing unfamiliar skills. The 'prac- 
tice' category was used to denote all other sport specific drills or activities. In the team sports this included strategy drills like offensive and defensive patterns in a half-court situation and individual skill drills such as passing or shooting in basketball and serving or setting in volleyball. Tennis and racquetball 'practice' activities were limited to drills on individual skills where the coach or another player would provide the opportunity to work on specific shots or covering certain areas of the court. General stretching exercises for warm-up, 'wind sprints', or other general training activities were not included.

A Sport Tester, model PE2000 was used to monitor the heart rates during the activities. This instrument has a digital display of heart rate based on the ECG signal from chest electrodes which is updated every five seconds. Every 30 seconds, the current five second heart rate value is put into memory and can be recalled and recorded after the monitoring session. The recorded heart rates thus represent a sample of the average heart rate over a five second interval taken every 30 seconds. Each instrument was checked for accuracy in the laboratory by comparison to a direct ECG recording, and with the exception of infrequent aberrant values the monitor values agreed with the ECG recording within \pm 2 beats/minute. Aberrant values during actual monitoring were identified and deleted by scanning the individual records for heart rates above 220 beats/min and/or a single value that was different from adjacent valves by more than 20 beats/min since these values reflected the aberrant valves noted during validification of the instrument.

Descriptive statistics were calculated for each individual including average heart rate, standard deviation and range of the heart rates, and the percentage of heart rate values greater than 140 beats/minute. The value of 140 beats/minute was selected on the basis of Knutsson, Lewenhaupt-Olsson and Thorsen, (1973) findings of a significant training effect in paraplegics with 140 as the minimal training heart rate. This value is estimated to be approximately $60 \%$ of the heart rate reserve of the present subjects. The average heart rate, standard error of the means, total range of heart rates, and average percentage above 140 beats/min. were calculated from the individual subject's values within each of the eight activity categories. Differences between the mean heart rates in each category were compared using a t-test with a 05 level of significance. The results of the t-tests, however, should be interpreted with caution due to the small sample size in some categories.

\section{Results}

Tables I to IV present the individual and group average heart rates for wheelchair basketball, volleyball, tennis, and racquetball, respectively. The two lowest heart rates (tennis and racquetball 'practice') were significantly lower than the three highest heart rates (basketball, racquetball, and tennis 'game'), and the volleyball practice value was lower than the basketball and racquetball 'game' averages. The only other statistically significant difference was the higher heart rate during the basketball 'game' condition in comparison to the volleyball 'game' value. The percentage of time when the average individual's heart rate was above 140 beats/minute varied from $0 \%$ for tennis and racquetball 'practice' to $62 \%$ for basketball 'game' conditions. There was, however, fairly large inter- 
Table I Heart rates during wheelchair basketball

\begin{tabular}{lccrc}
\hline & Average & S.D. & Range & \% Time > 140 \\
\hline & & Practice & & \\
Subject 1 & 143 & 17.4 & $97-170$ & 74 \\
Subject 2 & 142 & 16.9 & $105-170$ & 62 \\
Subject 3 & 111 & 16.0 & $86-141$ & 4 \\
Subject 4 & 93 & 13.0 & $76-117$ & 0 \\
Practice Totals & 122 & 12.3 & $76-170$ & 35 \\
& & Game & & \\
Subject 1 & 161 & 10.4 & $131-173$ & 91 \\
Subject 2 & 135 & 15.5 & $91-156$ & 46 \\
Subject 3 & 136 & 9.5 & $115-148$ & 43 \\
Subject 4 & 145 & 20.0 & $101-172$ & 67 \\
Subject 5 & 137 & 7.6 & $110-150$ & 33 \\
Subject 6 & 181 & 17.7 & $123-202$ & 94 \\
Subject 7 & 144 & 22.4 & $98-180$ & 59 \\
Game Totals & 148 & 6.4 & $91-202$ & 62 \\
\hline
\end{tabular}

Table II Heart rates during wheelchair volleyball

\begin{tabular}{lrccc}
\hline & Average & S.D. & Range & \% Time $>140$ \\
\hline & & Practice & & \\
Subject 1 & 97 & 6.6 & $83-108$ & 0 \\
Subject 2 & 106 & 7.0 & $91-119$ & 0 \\
Subject 3 & 83 & 7.9 & $68-100$ & 0 \\
Subject 4 & 121 & 11.2 & $97-153$ & 9 \\
Subject 5 & 112 & 18.0 & $68-145$ & 8 \\
Subject 6 & 123 & 12.9 & $104-142$ & 8 \\
Subject 7 & 99 & 5.0 & $89-108$ & 0 \\
Practice Totals & 106 & 5.4 & $68-153$ & 4 \\
& & & & \\
Subject 1 & 120 & Game & $95-139$ & 0 \\
Subject 2 & 131 & 9.5 & $105-149$ & 13 \\
Subject 3 & 93 & 13.1 & $78-134$ & 0 \\
Game Totals & 115 & 11.3 & $78-149$ & 4 \\
\hline
\end{tabular}

Table III Heart rates during wheelchair tennis

\begin{tabular}{lcccc}
\hline & Average & S.D. & Range & $\%$ Time > 140 \\
\hline & 90 & Practice & & \\
Subject 1 & 13.0 & $71-125$ & 0 \\
Subject 2 & 87 & 4.7 & $80-97$ & 0 \\
Practice Totals & 89 & 1.5 & $71-125$ & 0 \\
& & Game & & \\
Subject 1 & 121 & 13.0 & $88-133$ & 0 \\
Subject 2 & 127 & 8.0 & $107-136$ & 0 \\
Subject 3 & 135 & 10.5 & $103-145$ & 32 \\
Game Totals & 128 & 4.1 & $88-145$ & 11 \\
\hline
\end{tabular}


Table IV Heart rates during wheelchair racquetball

\begin{tabular}{lcccc}
\hline & Average & S.D. & Range & \% Time > 140 \\
\hline & & Practice & & 0 \\
Subject 1 & 106 & 7.4 & $88-122$ & 0 \\
Subject 2 & 98 & 5.7 & $80-106$ & 0 \\
Subject 3 & 104 & 6.9 & $82-127$ & 0 \\
Subject 4 & 76 & 9.9 & $60-98$ & 0 \\
Practice Totals & 96 & 6.9 & $60-127$ & \\
& & & & \\
Subject 1 & 137 & 12.0 & $108-156$ & 0 \\
Subject 2 & 124 & 7.7 & $110-138$ & 57 \\
Subject 3 & 141 & 8.1 & $116-151$ & 34 \\
Game Totals & 134 & 5.1 & $108-156$ & 34 \\
\hline
\end{tabular}

individual variability within each activity in average heart rate and the percentage of heart rates above 140 beats/minute. This reflects both the variable intensity of different activities or time periods within each category and the effort of the subject in the activity. Basketball 'practice' and basketball and racquetball 'game' conditions exhibited an inter-subject difference of over $50 \%$ in percentage of time above 140 beats/minute, while tennis and racquetball 'practice' did not elicit any heart rates above 140 in any subject. At least one subject spent $25 \%$ or more of his time with a heart rate greater than 140 beats/ min. in only the basketball 'game' and 'practice', racquetball 'game', and tennis 'game' conditions.

\section{Discussion}

The data presented provide an index of the exercise intensity of participants in the wheelchair sports monitored. The average basketball 'game' heart rate of 148 beats/minute is similar to the individual value of 144 beats/minute reported by Hjeltnes and Vokac (1979) and data on three players during a two game tournament (Dawson, William and Rape, 1980). The other team sport, volleyball, had the lowest average heart rate while tennis and racquetball had intermediate values. This is not a surprising result when considering the amount and duration of locomotor activity involved in each of these sports. That is, volleyball involves quick movements over a relatively short distance for an individual to cover his/her area of the court, tennis and racquetball involve covering somewhat longer distances, and basketball even longer as play moves from one end of the gym to the other. The 'game' heart rates, thus, appear generally related to the amount of wheelchair propulsion involved in the activity rather than the intensity of the specific skills of the sport. Hjeltnes and Vokac's (1979) finding of average heart rates in the 90 's with $0 \%$ above the $50 \%$ level of heart rate reserve during wheelchair table tennis seems consistent with this line of reasoning.

As noted, the 'practice' times tended to elicit lower heart rates than 'game' conditions within each sport, but firm conclusions are not warranted due to the small sample size in some sports. The most logical explanation for this trend is related to the amount of change in wheelchair propulsion from 'game' to 'practice' conditions. In racquetball and tennis, 'practice' conditions usually meant 
that the athlete was relatively stationary and would work on a particular stroke or shot with the ball set-up to accomplish this, or two players would rally, which involved hitting the ball toward rather than away from the opponent.

The wide between subject variability in percentage of heart rates above 140 beats/minute in basketball 'practice' appeared to reflect the amount of locomotion in the activity. Subjects 1 and 2 were involved in drills such as fast breaks, lay up shooting, dribbling, and passing which required movement up and down the gym floor, while subjects 3 and 4 participated in non-pressure drills such as perimeter shooting and half-court practice of offensive and defensive patterns. The subjects with higher heart rates and percentage of time greater than 140 beats/minute in the tennis (\#3) and racquetball (\#1 and 3) 'game' condition were generally involved in longer rallies for points than the other subjects which may reflect a higher skill level or their intensity of effort in going for the ball. There were no apparent explanations for the between subject variability within other activities, but limited notes were made about the activity during a monitoring session.

The minimal combination of intensity and duration of training which will produce improvement in the aerobic power of paraplegics has not been firmly established. Knutsson, et al., (1973) noted an improved PWC 170 following 6 weeks of an interval training regimen which elicited heart rates of 140-180 for a total of about 6 minutes during 4-5 days per week, while Pollock, et al., (1974) found a significant increase in maximal oxygen uptake following 20 weeks of arm cranking for 30 minutes, 3 days per week at a heart rate close to 160 beats/minute.

The monitored heart rates during wheelchair sports in the present study suggest that the average participant in wheelchair basketball and racquetball and some individuals in tennis would experience an aerobic training effect if participation was on a regular basis. Wheelchair volleyball, however, does not appear to elicit high enough heart rates in any of the subjects to be considered a possible aerobic training activity. It should be emphasised that any individual wishing to use participation in any sport as the basis of an aerobic fitness program should monitor their heart rate due to the noted large variability in individual responses.

\section{Acknowledgements}

The author wishes to express appreciation to the members of the Canadian Wheelchair Sports Association-B.C. Division for their cooperation, and to Jan Stogryn, Dianne Pendray, and Patti Woods for their technical assistance.

This study was supported by a grant from Fitness and Amateur Sport Canada.

\section{References}

BIERING-SORENSEN, F 1980 Classification of paralysed and amputee sportsmen. In H. Natvig (Ed.) Proceedings of the First International Medical Congress on Sports for the Disabled (pp. 44-54). Oslo; Royal Ministry of Church and Education, State Office for Youth and Sports. Coutts, KD, RHODEs, EC, MCKenZIE, DC 1983 Maximal exercise responses of tetraplegics and paraplegics. Fournal of Applied Physiology 55:479-482.

Dawson, GA, WILliaM, SG, RAPE, SM 1980 Heartrate and athletics. Sports 'N Spokes 6(1):1314. 
Di CARLo, SE, SuPp, MD, TAYLOR, HC 1983 Effect of arm ergometry training on physical work capacity of individuals with spinal cord injuries. Physical Therapy 63:1104-1107.

HJELTNES, N, VoKaC, Z 1979 Circulatory strain in everyday life of paraplegics. Scandinavian Fournal of Rehabilitation Medicine 11:67-73.

Hullemann, KD, List, M, Mathes, D, et al., 1975 Spiroergometric and telemetric investigations during the XXI International Stoke Mandeville Games 1972 in Heidelberg. Paraplegia 13:109123.

Knutsson, E, Lewenhaupt-Olsson, E, Thorsen, M 1973 Physical work capacity and physical conditioning in paraplegic patients. Paraplegia 11:205-216.

Mollinger, LA, SPURR, GB, GHATIT, AZ EL, et al., Daily energy expenditure and basal metabolic rates of patients with spinal cord injury. Archives of Physical Medicine and Rehabilitation 66:420-426.

Pollock, ML, Miller, HS, Linnerud, AC, et al., 1974 Arm pedaling as an endurance training regimen for the disabled. Archives of Physical Medicine and Rehabilitation 55:418-424. 